\title{
Large-scale disturbances preceding a fast halo CME
}

\author{
W.-G. Zong \\ National Center for Space Weather, China Meteorological Administration, Beijing 100081, PR China \\ e-mail: wgzong@nsmc.cma.gov.cn
}

Received 30 August 2007 / Accepted 10 December 2007

\begin{abstract}
Aims. The nature of coronal waves, often termed "EIT waves" is still unclear. Therefore new efforts are needed to investigate largescale disturbances during solar eruptions. In this paper, we present observations of an event occurring on 19 January 2005, and study the large-scale disturbance during the event in detail.

Methods. Using the high cadence images from SXI onboard GOES-12, the large-scale disturbance is identified. Combined with EIT $195 \AA$ images, TRACE UV $1600 \AA$ Amages, RHESSI hard X-ray data and MDI/SOHO longitudinal magnetic field maps, the intrinsic process of the large-scale disturbance is discussed.

Results. The large-scale disturbance propagated over a distance about $3.8 \times 10^{5} \mathrm{~km}$ at a velocity of about $390 \mathrm{~km} \mathrm{~s}^{-1}$. Along the trajectory of the disturbance, a brightening line was left behind, which was coincident with the dense structure in EIT intensity images. With the morphology and metric radio spectrum data, it was found that the disturbance was not a wave but the low-coronal signatures of the eruption.
\end{abstract}

Key words. Sun: flares - Sun: coronal mass ejections (CMEs) - Sun: magnetic fields

\section{Introduction}

During large eruptions, such as coronal mass ejections (CMEs) or flares, large-scale disturbances are usually observed propagating out into the magnetized solar atmosphere. According to the observation wavelengths, these disturbances appear as different signatures.

In chromospheric images recorded in the wings of $\mathrm{H} \alpha$, the disturbances often appear as Moreton waves, seen as bright arclike fronts propagating away from the site of solar flare. They propagate over distances in the order of $5 \times 10^{5} \mathrm{~km}$ with velocities ranging from 500 to $2000 \mathrm{~km} \mathrm{~s}^{-1}$ (Moreton \& Ramsey 1960). When they encounter a filament, they may induce oscillations in the filament, and as a result, the filament periodically disappears and reappears in the $\mathrm{H} \alpha$ line-center images (Smith \& Harvey 1971). Therefore "winking" filaments are usually considered to be the signatures of Moreton waves and are used to measure the velocities of Moreton waves (Okamoto et al. 2004). Given that it is hard to explain chromospheric Moreton waves as shock waves propagating in the chromosphere itself, Uchida (1968) interpreted them as skirts of fast mode coronal MHD waves sweeping over the chromosphere.

Large-scale disturbances are also observed as coronal signatures. With the observations of the Extreme-Ultraviolet Imaging Telescope (EIT, Delaboudinière et al. 1995) on board the Solar and Heliospheric Observatory (SOHO) spacecraft, large-scale disturbances appearing as semi-circular diffuse emission enhancements followed by expanding "dimming regions" are usually found to propagate outward from the flaring active region (Moses et al. 1997; Thompson et al. 1999). In some cases, these disturbances were observed in coincidence with Moreton waves (Thompson et al. 2000; Pohjolainen et al. 2001; Warmuth et al. 2001), therefore they were considered as the coronal counterparts of Moreton waves, and labeled as "EIT waves". However, in the statistical study of EIT waves, it has been reported that the velocities of EIT waves were $170-350 \mathrm{~km} \mathrm{~s}^{-1}$ (Klassen et al. 2000), which were much less than the velocities of Moreton waves. Delannée \& Aulanier (1999) found EIT waves stopped near the separatrix between active regions and thus appeared as a stationary front. Harra \& Sterling (2003) presented a event with two wave-like signatures observed in EUV lines images. They both suggested that the EIT waves were associated with CMEs and resulted from the magnetic field evolution involved in CMEs. Using numerical simulations, Chen et al. $(2002,2005)$ proposed a model to reconcile the discrepancy between EIT waves and Moreton waves and to explain the stationary front. In their model, EIT waves were described as the successive stretching or opening of closed field lines driven by an erupting flux rope.

As for EIT "waves", it is still being debated whether they are true waves or not. To understand this, the observations in other temperatures are also included. Vršnak et al. (2002) reported wave signatures observed in He I (10830 A) filtergrams. Although the formation mechanism of $\mathrm{He}$ I line is complicated in a solar atmosphere, the coincidences have been found between the chromospheric waves observed in this line and the coronal waves (Gilbert et al. 2004). Warmuth et al. (2005) presented the coronal signatures observed with the Solar X-Ray Imager (SXI) onboard the Geostationary Operational Environmental Satellites (GOES) satellite. By comparing the wave signatures with EIT, $\mathrm{H} \alpha$, and $\mathrm{He} \mathrm{I}$ data, these signatures were identified as waves, which propagated at decelerations of several hundred meters per second per second. These decelerations along with the low time cadence of the EIT instrument were considered as the cause of the velocity discrepancy between EIT waves and chromospheric Moreton waves (Warmuth et al. 2004). 

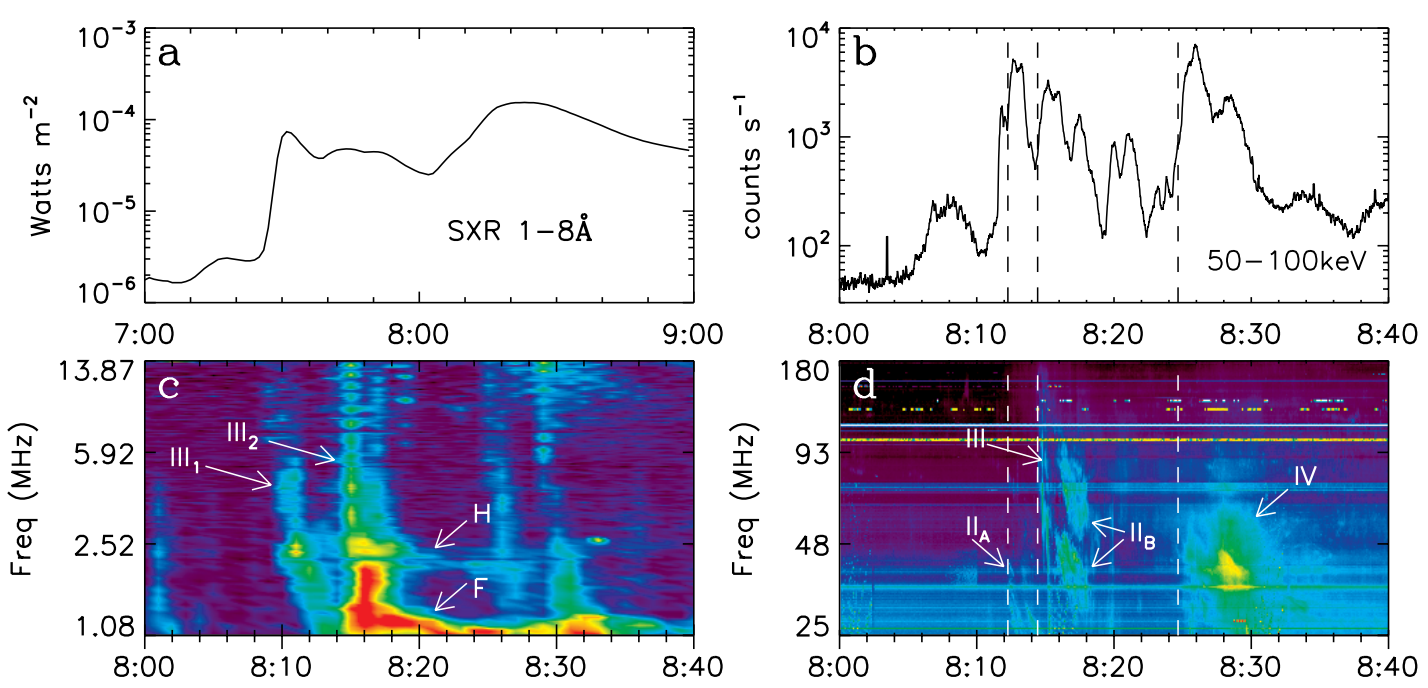

Fig. 1. a) Soft 1-8 $\AA$ X-ray flux obtained by GOES-10. b) Hard X-ray light curve from RHESSI, the energy band is $50-100 \mathrm{keV}$. c) Radio dynamic spectrum from WAVES/WIND. The frequency range is from 1.075 to $13.875 \mathrm{MHz}$. d) Radio dynamic spectrum from RSTN. The frequency range is from 25 to $180 \mathrm{MHz}$.

In fact, EIT "waves" show a broad range of morphological characteristics and velocities (Biesecker et al. 2002), and they may be caused by different processes (Zhukov \& Auchère 2004). Some of them are true waves but the others are not. In this study, we present the detailed observations of an event, during which a large-scale coronal disturbance was found propagating over a long distance. With the high time cadence images obtained by SXI onboard GOES-12, signatures of the disturbance can be identified sequentially. Along with EUV, radio and hard X-Ray data, the physical nature of the disturbance is studied. The paper is organized in the following way. Data sources are introduced in Sect. 2, observations are described in detail in Sect. 3, and Sect. 4 is dedicated to the discussion.

\section{Data sources}

The images obtained by SXI onboard GOES-12 are used to find the evolution of the large-scale disturbance. These images were acquired with the thin polyimide filter (PTHN) covering temperatures of about $4 \mathrm{MK}$, which has a broad temperature response of about \pm 2 MK FWHM. The exposure time is $3 \mathrm{~s}$. The SXI has a low spatial resolution of about $5^{\prime \prime}$ per pixel, but it is enough to identify large-scale signatures. With the high time cadence of about 2 min per image, the large-scale disturbance can be traced sequentially. EIT full-disk Fe XII $195 \AA$ A images are also used. Both SXI and EIT images show coronal signatures which appear similar in some ways, but since EIT has a better spatial resolution of 2.6" per pixel, it reveals the signatures more clearly.

Both metric and decametric-hectometric (DH) radio dynamic spectrums are presented in this study, to check the relationship with the large-scale disturbance. The metric radio dynamic spectrum, ranging from 25 to $180 \mathrm{MHz}$, was obtained by the solar radio spectrograph located at Learmonth in Australia, which is one of the instruments of the Radio Solar Telescope Network (RSTN). It has a temporal resolution of about $3 \mathrm{~s}$. The DH radio dynamic spectrum with a frequency range of $1.075-13.875 \mathrm{MHz}$ and a temporal resolution of about 1 min, was observed by the (radio and plasma) WAVES experiment (Bougeret et al. 1995) on the Wind spacecraft.

A Hard X-ray (HXR) light curve from the Reuven Ramaty High Energy Solar Spectroscopic Imager (RHESSI) is used in the 50-100 keV energy band. The temporal resolution is $1 \mathrm{~s}$. Soft 1-8 $\AA$ X-Ray flux is used to identify flares, it was obtained by GOES-10 with a temporal resolution of $1 \mathrm{~min}$.

By the Pixon reconstruction algorithms, we got HXR images taken by RHESSI. Transition Region and Coronal Explorer (TRACE) UV $1600 \AA$ images are used to be co-aligned with the HXR images. Although the TRACE observations were absent during the HXR bursts, the flare ribbons were relatively stationary during the eruption, so we select images near the HXR eruptive period in the co-alignment. The Michelson Doppler Imager (MDI, Scherrer et al. 1995) onboard the SOHO satellite provided the longitudinal magnetic field map. The uncertainty of the measurement is about 10 Gauss. The pixel size is $1.97^{\prime \prime}$.

\section{Observations}

\subsection{Description of the flare/CME event}

On 19 January 2005, big flares were successively observed from 07:30 to 08:30 UT. It was found that they were from an identical active region labeled as NOAA0720. When the flares burst, the active region was located at around $\mathrm{N} 16^{\circ} \mathrm{W} 53^{\circ}$. The soft $1-8 \AA$ $\mathrm{X}$-ray (SXR) flux is presented in Fig. 1a. The SXR flux increased rapidly at 07:25 UT, and reached the first peak at about 07:31 UT, which was classified as M6.7. The second rapid increase of the SXR flux occurred at 08:03 UT, and was caused by another flare. It reached a peak at 08:22 UT and was classified as X1.3. The large-scale disturbance and the fast halo CME were mainly associated with this flare.

Hard X-ray and radio emissions accompanied with the X1.3 flare are indicated in Figs. 2b-d. The HXR light curve shows multiple bursts. The first burst started at 08:04 UT and peaked at 08:08 UT. Its peak counting rate was 300 counts $\mathrm{s}^{-1}$. The duration of this weak burst was about $6 \mathrm{~min}$, it ended at 08:10 UT. During this period, both the DH and the metric radio emissions were relatively quiet, only some faint bursts could be found. After the first burst, the HXR light curve became more noisy. There were multiple bursts with the peak counting rate over 1000 counts $\mathrm{s}^{-1}$. Simultaneously, multiple radio bursts occurred in the $\mathrm{DH}$ and the metric radio spectrums. At about 08:10 UT and 08:14 UT, as indicated in Fig. 1c, type III radio 

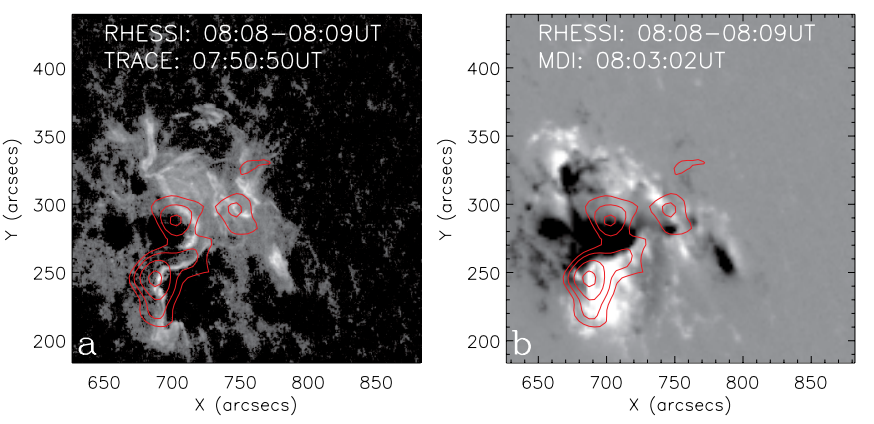

Fig. 2. a) Trace $1600 \AA ̊$ image at 07:50:50 UT. b) Longitudinal magnetic map obtained by MDI onboard SOHO at 08:03:02 UT. The magnetic field scale is confined to -300 to 300 Gauss. The RHESSI HXR intensity image in 50-100 KeV energy band is co-aligned as red contour lines, the contour levels are $0.27,0.4,0.6$ and 0.9 of maximum intensity.

bursts labeled as " $\mathrm{III}_{1}$ " and " $\mathrm{III}_{2}$ " were found with the frequencies drifting from the upper limit to the lower limit of the spectrum range. At about 08:14 UT, a DH type II radio burst started. It appeared as harmonic and fundamental drifting bands. The starting frequency of fundamental band was about $1.38 \mathrm{MHz}$, at 08:28 UT, it drifted out of the spectrum range, the frequency drifting rate was about $21 \mathrm{kHz}$ min $^{-1}$. The starting frequency of harmonic band was about $2.72 \mathrm{MHz}$, it stopped at 08:28 UT, the frequency drifting rate was about $40 \mathrm{kHz} \mathrm{min}^{-1}$.

Metric radio bursts occurred mainly after 08:12 UT. As indicated by vertical dash lines in Fig. 1d, the metric radio bursts could be separated into three parts. The first part started at 08:12 UT, and was composed of a type II radio burst (labeled as " $\mathrm{II}_{\mathrm{A}}$ ") appearing as a split band, which started at a frequency of about $42 \mathrm{MHz}$ with the frequency drifting rate of about $11.3 \mathrm{MHz} \mathrm{min}^{-1}$. About one and a half minutes later, it drifted out of the lower limit of the spectrum range. The second part was composed of a type III and a type II radio bursts. It starts at 08:14 UT. The frequency of the type III radio burst drifted from the upper limit to the lower limit of the spectrum range. It appeared to be related with the type III radio burst labeled as " $\mathrm{III}_{2}$ " in DH spectrum. They might be two parts of a single burst process. The type II radio burst labeled as " $\mathrm{II}_{\mathrm{B}}$ " appeared as two drifting bands. The starting frequencies were $75 \mathrm{MHz}$ and $150 \mathrm{MHz}$ respectively. They both stopped at 08:18 UT, the frequency drifting rate of fundamental band was about $15.7 \mathrm{MHz} \mathrm{min}^{-1}$. The third part was a type IV radio burst starting at 08:24 UT. By comparing metric radio bursts with the HXR light curve, it seemed that there was a temporal correlation between the strong hard X-ray bursts and metric radio bursts. The three parts metric radio burst corresponded to the strong HXR bursts with the peak value over 2000 counts s $^{-1}$.

Figure 2 shows the topology of the flare. The TRACE UV $1600 \AA$ image indicated that this was a multi-ribbons flare. The over-plotted RHESSI intensity contours exhibited that there were four HXR kernels, which were located at four different ribbons. With the longitudinal magnetic field map obtained by MDI onboard SOHO, it was found that the magnetic configuration was very complex. There were two big polarities located at the lower left part of Fig. 2b, with some small patches located around them. Correspondingly, the left two HXR kernels lay on the major polarities of the active region, and the right two, appearing fainter, lay on the magnetic patches around the active region. Among the four HXR kernels, two were located in positive and the other two in negative magnetic polarity, which implied that the flare was in a quadrupolar magnetic topology.
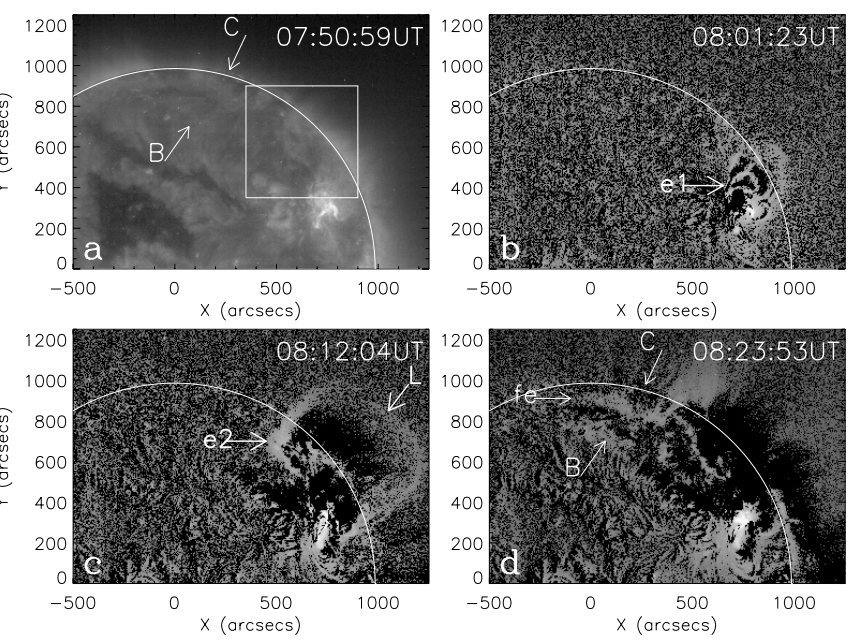

Fig. 3. a) EIT $195 \AA$ intensity image at 07:50:59 UT. The location of the polar coronal hole is indicated as " $\mathrm{C}$ " and the boundary of the filament channel is indicated as " $\mathrm{B}$ ". The white rectangle indicates the area which is indicated in Fig. 5. b)-d) EIT $195 \AA$ base difference images at 08:01:23 UT, 08:12:04 UT and 08:23:53 UT, the image at 07:50:59 UT has been subtracted. The arrows "e1-e2" point to the footpoints of the loop-like eruptive features (in b) and c)), "L" indicates the well defined loop hanging out of the solar limb (in c) and "fe" is the extreme extent point of the diffuse emission enhancements on the boundary of the polar coronal hole (in d)).

EIT images are displayed in Fig. 3. The EIT intensity image at 07:50:59 UT showed the coronal environment around the active region prior to the eruption. There was a coronal hole near the north pole. To the left of the eruption region, there was a filament channel. With the difference image at 08:01:23 UT, it was found that there were loop-like structures stretching out, of which the left footpoint is indicated as "e1". At 08:12:04 UT, a well defined loop was found to hang out of the solar limb with the left footpoint indicated by "e2" and the top indicated by "L". There was a dimming region behind the loop, which also expanded out of the solar limb. The loop was not centric at the flare site on the solar surface, but to the upper left of it. At the left foot of the loop, there was a brightening line. It extended to the flare site, but it didn't connect with the flare site, i.e. there was a gap (see also Fig. 5c). At 08:23:53 UT, the well defined loop erupted, with only dimming region left behind. Diffuse emission enhancements occurred on the northern boundary of the polar coronal hole and the upper right boundary of the filament channel. At 08:29 UT, the front of a fast halo CME was observed in the Large Angle and Spectrometric Coronagraph (LASCO, Brueckner et al. 1995) C2 image (see http: //cdaw.gsfc.nasa.gov/CME_list), and from then on the CME propagated out at a speed about $2020 \mathrm{~km} \mathrm{~s}^{-1}$ with a deceleration about $43.8 \mathrm{~m} \mathrm{~s}^{-2}$.

\subsection{Large-scale disturbance}

With the SXI running difference images, the signatures of the large-scale disturbance were identified sequentially in Fig. 4. At first, the disturbance seemed to be confined to the flare site. At 08:00:51 UT, the eruptive structures labeled as " $\mathrm{t} 1$ " and " $t 2$ " were found to move upward. Compared with Fig. 3b, the eruptive structures were coincident with the loop-like structures stretching out in the EIT image. At 08:02:29 UT, the left part of " $t 1$ " became straighter and the top of " $\mathrm{t} 2$ " ascended, which implied that the eruptive structures kept moving upward. At 

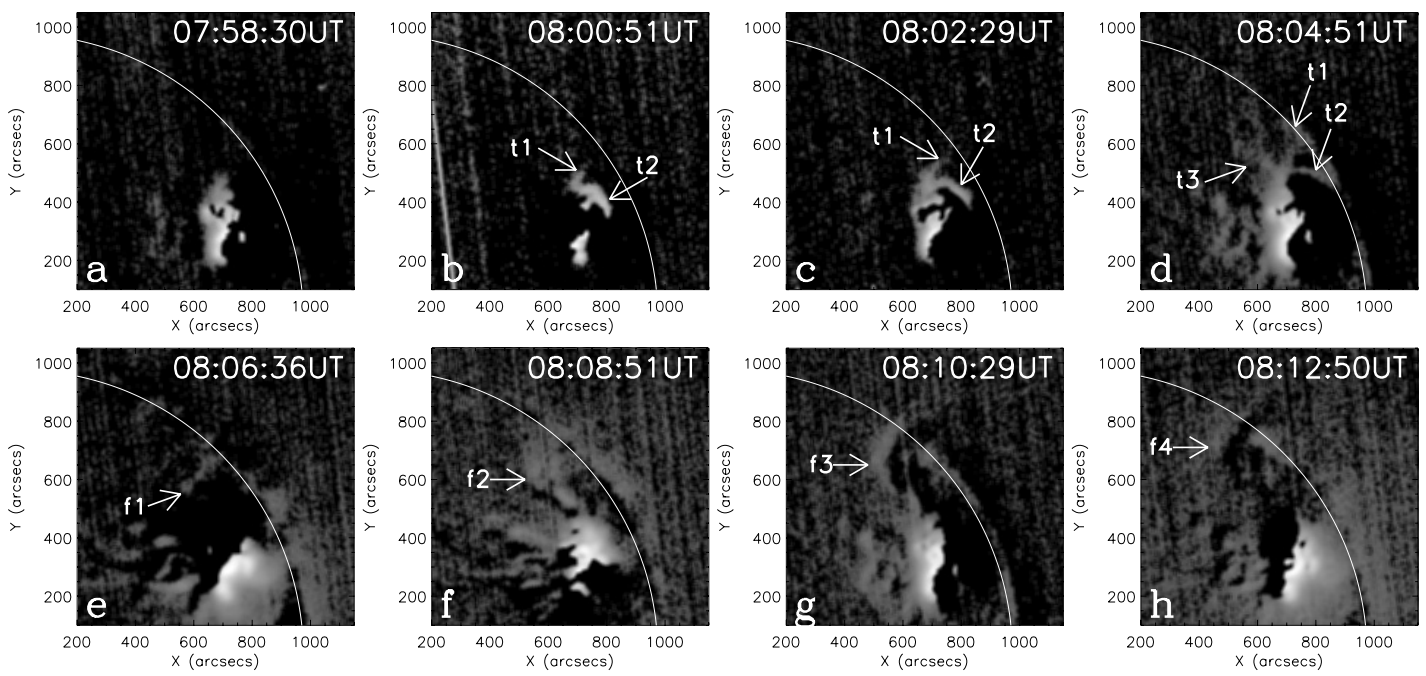

Fig. 4. Sequence of SXI running difference images. The eruptive features from the flare site are indicated by "t 1 ", "t2" and "t 3 ". The points indicated by "f1-f4" are the footpoints of the coronal bright signatures on the solar surface.

08:04:51 UT, the top of "t2" move to a higher altitude, and the left part of " $t 1$ " came to a vertical position. To the left of " $t 1$ ", diffuse emission enhancements labeled as " $\mathrm{t} 3$ " showed up. From then on, the signatures of the disturbance occurred sequentially to the northeast of the flare. At 08:06:36 UT, the disturbance appeared as a thin white linear structure, and at 08:08:51 UT, it became diffuse, some brightening patches were found to extend to the solar limb, the trajectory of the disturbance appeared as a brightening line connecting to the flare site. At 08:10:29 UT and 08:12:50 UT, the signatures of the disturbance looked like left foot of the well defined loop as shown in Fig. 3c (see also Fig. 5c). As they were contaminated by the halo brightening of the flare, the signatures of disturbances were difficult to recognize in the following sequence SXI images. In Figs. $4 \mathrm{e}-\mathrm{h}$, the farthest brightening points in the feet (considered as the parts on the solar surface) of the signatures were identified as "f1", "f2", "f3" and "f4". The distance of the point " 44 " from the flare site was estimated to be $3.8 \times 10^{5} \mathrm{~km}$.

Figure 5a indicates the longitudinal magnetic field map in the propagation direction of the disturbance. It was found that the magnetic fields were weak along the disturbance propagation direction, with only minor weak polarities lying there. The magnetic field strength of these minor polarities was less than 50 Gauss. Compared with the EIT intensity image taken at 08:01 UT before the onset time of the flare, these minor weak polarities were correlated with bright signatures, especially around "f1", "f2" and "f3", there was a long bright structure, which extended out from the flare site and stopped near "f3". This structure could be separated into two parts. The first part was from " 11 " to "12", this part appeared thin and smooth, it looked like a loop. The points " 11 " and " 12 " were endpoints of the loop. The endpoint "12" was easy to recognize, considered the projection effect, and was located at a minor positive polarity. However, because there were other loops around " 11 ", the location of this endpoint was not easy to identify, and maybe the real endpoint was hidden behind the above loops. The second part of the long bright structure was from " 12 " to "f3", with this part appearing thick and diffuse. In fact the bright structure could even be found to extend from "f3" to upper left, but was much fainter. Around the points "e2" and "f4", there were only faint bright points.

Figure $5 \mathrm{c}$ shows the EIT base difference image at 08:12:04 UT, the disturbance propagated to the distance between
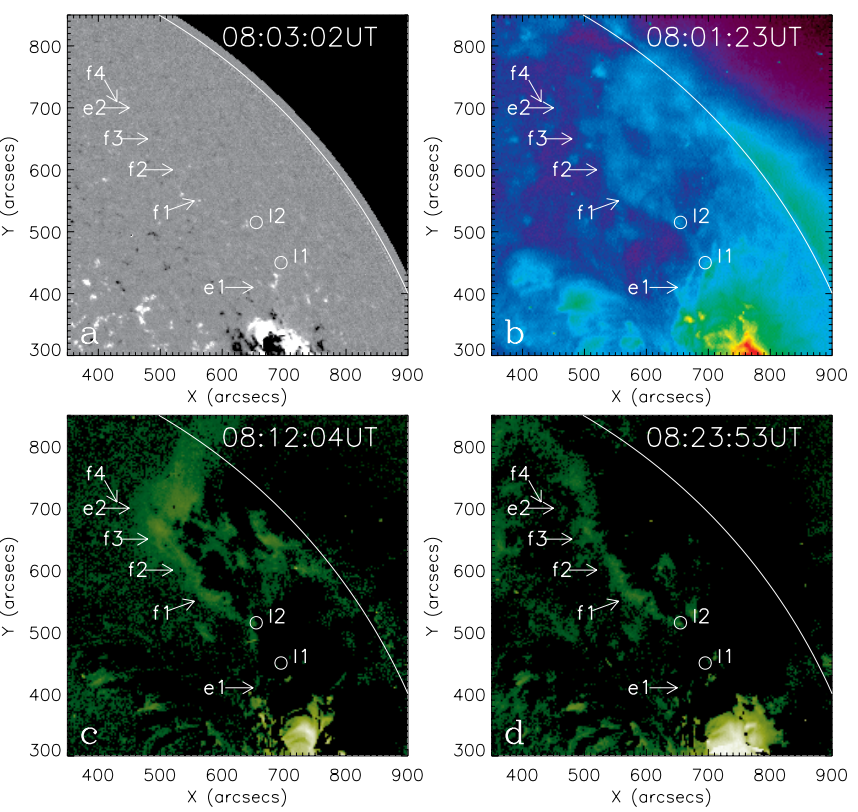

Fig. 5. a) The longitudinal magnetic field map at 08:03:02 UT, the magnetic field strengths are confined in -50 to 50 Gauss. b) EIT intensity image at 08:01:23 UT. c), d) EIT base difference images at 08:12:04 UT and 08:23:53 UT, the image at 07:50:59 UT has been subtracted. The points "f1-f4" are the same as indicated in Fig. 4 and "e1-e2" are the same as indicated in Fig. 3.

"f3" and "f4" (see also Fig. 3c). The bright structure between "11" and "12" partly disappeared, and as a result, dimming was left between these two points, and brightening points occurred near "11" and "12". Because the loops above "11" also erupted, dimming was found around " 11 ". There was a brightening line connecting "12" to "e2", and the points "f1", "f2" and "f3" were lying along it. As indicated in the base difference EIT image at 08:23:53 UT, the bright line extended to the boundary of the polar coronal hole, but the part between " 12 " and "e2" became thinner.

The distances of the footpoints of the disturbance from the flare site are shown in Fig. 6, which were obtained by taking the sun as a sphere. From the result, we found the disturbance 


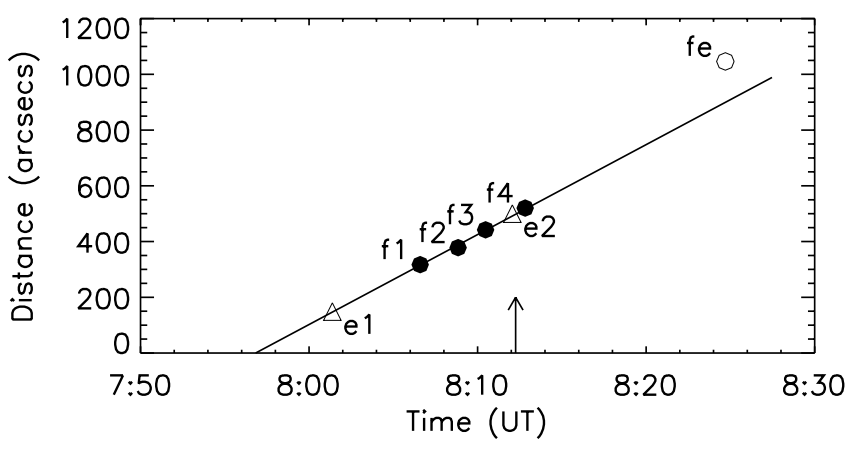

Fig. 6. Distances of the footpoints of the brightening signatures from the flare site. The black arrow shows the onset time of metric radio bursts.

before 08:13 UT propagated at an almost constant velocity. By the linear least square fit method, the velocity was derived, which was about $390 \mathrm{~km} \mathrm{~s}^{-1}$. However, "fe" lied above the fit line. This indicated the disturbance propagated out at a much higher mean speed after 08:13 UT. Using two points "e2" and "fe", the estimated speed was about $540 \mathrm{~km} \mathrm{~s}^{-1}$. The footpoints "e1-e2" and "f1-f4" fitted in a line, which indicated there was a coincidence between the SXI and the EIT images. The black arrow in Fig. 6 exhibited the onset time of metric radio bursts. Until the disturbance propagated to "e2", the metric radio spectrum was relatively quiet.

\section{Discussion}

The signatures of the disturbance in the event can be traced sequentially in SXI images. They were found to propagate in a narrow angle. They propagated through the corona over a distance of about $3.8 \times 10^{5} \mathrm{~km}$ at a velocity of about $390 \mathrm{~km} \mathrm{~s}^{-1}$. The distance and the velocity are in the range of common EIT "waves" (Klassen et al. 2000). However, the observations indicated they were not waves. By comparing Figs. $3 g-h$ with Fig. $4 d$, it was found that "f3" and " $f 4$ " were located near the foot of a well defined loop, which hangs out of the solar limb. It seemed that there was no wave disturbance other than the low-coronal signatures of the eruption.

Since the metric radio spectrum was relatively quiet until the disturbances propagated to the point "e2", the described disturbance was not related to the metric radio burst shock. Furthermore, the metric radio bursts showed a temporal relationship with HXR bursts, implying that the metric radio bursts were closely related to magnetic reconnection in the flare site, and that they might be flare-induced (Cane \& Erickson 2005; Vršnak et al. 2006). At the moment when the metric type II radio bursts started, the disturbance had propagated to a distance far away from the flare site, therefore the disturbance could not be the metric type II burst shock induced by flare. However, "fe" and the diffuse emission enhancements on the boundary of the filament channel were ahead of the eruption, they could be a flank of the shock driven by CME which at a larger height might have caused DH type II radio bursts, so the relation between the disturbance and DH type II shock could not be excluded.

In fact, we prefer treating the disturbance as evolution of magnetic fields. The model of Attrill et al. (2007) can be applied to interpret the disturbance (see Fig. 4 in their paper). The loop-like structures " $t 1$ " and " 2 " in Figs. $4 b-d$ could be taken as the expanding CME magnetic fields, the structure between "11" and "12" in Fig. 5 could be taken as a quiet-sun (QS) loop. The reconnection between the expanding CME magnetic fields and the QS loop caused the brightening signatures at the endpoints " 11 " and " 12 ", and dimming between them and around "11". The dense structure between "12" and "f4" might consist of many low-lying magnetic field lines connecting weak-field regions, which could act as QS loops. As the CME magnetic field lines stretched out, magnetic reconnection progressed sequentially between the CME magnetic field lines and these low-lying magnetic field lines. As a result, the left feet of CME magnetic field lines swept along the dense structure between "12" and " 4 " and left a brightening line along the trajectory. Simultaneously, dimming occurred behind the CME magnetic field lines due to the expanding volume.

As indicated in Fig. 2, the first stage of the flare was in a quadrupolar magnetic topology, the flare could be induced by the loop-loop interactions. This was different to the model proposed by Chen et al. (2005), in which the eruption was caused by an ejected flux rope. However, the model of Delannée (2000) might be a choice to interpret the flare, but it was difficult to explain the propagation of the bright front with it.

Neupert (1989) has presented direct evidence of the interactions between the lower corona and the chromosphere. There might be chromospheric counterpart of the coronal disturbance. Tang \& Moore (1982) have presented two flares with $\mathrm{H} \alpha$ emission patches in remote quiet regions more than $10^{5} \mathrm{~km}$ away from the flare site. They explained the observations as energized electrons traveling in closed magnetic loops connecting the flare site to the remote patches. Balasubramaniam et al. (2005) reported a phenomena called sequential chromospheric brightenings (SCBs), which were observed to propagate away from the flare site at a velocity of $600-800 \mathrm{~km} \mathrm{~s}^{-1}$. They were quite different to Moreton waves, and the authors treated them as the result of a sequential tearing away of coronal field lines during a CME. Liu et al. (2006) studied the remote brightenings in multiple wavelengths, and found the brightenings were resulting from hot particles transporting through large-scale loops. In these studies, SCBs exposed some features similar to the disturbance described in this study, such as narrow propagation angle and loops eruption in EIT images. In future work, with the coronal and the chromospheric observations both in high cadence, the connection between the corona and the chromosphere may be understood more clearly.

Acknowledgements. We greatly appreciate the anonymous referee for very helpful comments and suggestions. The authors are also grateful to the SOHO/EIT, SOHO/MDI, SOHO/LASCO, GOES, WIND/WAVES, TRACE, RHESSI and RSTN teams for their open data policy. We used the data from SOHO CME catalog. This CME catalog is generated and maintained at the CDAW Data Center by NASA and The Catholic University of America in cooperation with the Naval Research Laboratory. SOHO is a project of international cooperation between ESA and NASA.

\section{References}

Attrill, G. D. R., Harra, L. K., van Driel-Gesztelyi, L., \& Démoulin, P. 2007, ApJ, 656, L101

Balasubramaniam, K. S., Pevtsov, A. A., Neidig, D. F., et al. 2005, ApJ, 630, 1160

Biesecker, D. A., Myers, D. D., Thompson, B. J., Hammer, D. M., \& Vourlidas, A. 2002, ApJ, 569, 1009

Bougeret, J.-L., Kaiser, M. L., Kellogg, P. J., et al. 1995, Space Sci. Rev., 71, 231 Brosius, J. W., \& Holman, G. D. 2007, ApJ, 659, L73

Brueckner, G. E., Howard, R. A., Koomen, M. J., et al. 1995, Sol. Phys., 162, 357 
Cane, H. V., \& Erickson, W. C. 2005, ApJ, 623, 1180

Chen, P. F., Wu, S. T., Shibata, K., \& Fang, C. 2002, ApJ, 572, L99

Chen, P. F., Shibata, K., \& Fang, C. 2005, ApJ, 622, 1202

Delaboudinière, J.-P., Artzner, G. E., Brunaud, J., et al. 1995, Sol. Phys., 162, 291

Delannée, C. 2000, ApJ, 545, 512

Delannée, C., \& Aulanier, G. 1999, Sol. Phys., 190, 107

Gilbert, H. R., Holzer, T. E., Thompson, B. J., \& Burkepile, J. T. 2004, ApJ, 607, 540

Harra, L. k., \& Sterling, A. C. 2003, ApJ, 587, 429

Klassen, A., Aurass, H., Mann, G., \& Thompson, B. J. 2000, A\&AS, 141, 357

Liu, C., Lee, J., Deng, N., Gary, D. E., \& Wang, H. M. 2006, ApJ, 642, 1205

Moreton, G. E., \& Ramsey, H. E. 1960, PASP, 72, 357

Moses, D., Clette, F., Delaboudinière, J.-P., et al. 1997, Sol. Phys., 175, 571

Neupert, W. M. 1989, ApJ, 344, 504

Pohjolainen, S., Maia, D., Pick, M., et al. 2001, ApJ, 556, 42
Scherrer, P. H., Bogart, R. S., Bush, R. I., et al. 1995, Sol. Phys., 162, 129 Smith, S. F., \& Harvey, K. L. 1971, in Physics of the Solar Corona, ed. C. J. Macris (Dordrecht: Reidel), 156

Okamoto, T. J., Nakai, H., Keiyama, A., et al. 2004, ApJ, 608, 1124

Tang, F., \& Moore, R. L. 1982, Sol. Phys., 77, 263

Thompson, B. J., Gurman, J. B., Neupert, W. M., et al. 1999, ApJ, 517, L151

Thompson, B. J., Reynolds, B., Aurass, H., et al. 2000, Sol. Phys., 193, 161

Uchida, Y. 1968, Sol. Phys., 4, 30

Uchida, Y. 1974, Sol. Phys., 39, 431

Vršnak, B., Warmuth, A., Brajša, R., \& Hansimeier, A. 2002, A\&A, 394, 299

Vršnak, B., Warmuth, A., Temmer, M., et al. 2006, A\&A, 448, 739

Warmuth, A., Vršnak, B., Aurass, H., \& Hanslmeier, A. 2001, ApJ, 560, L105

Warmuth, A., Vrs̆nak, B., Magdalenić, J., Hanslmeier, A., \& Otruba, W. 2004, A\&A, 418, 1101

Warmuth, A., Mann, G., \& Aurass, H. 2005, ApJ, 626, L21

Zhukov, A. N., \& Auchère, F. 2004, A\&A, 427, 705 